
Some deep inoculations were made by passing the virus
through a wound several millimetres in extent, made hy a lancet. The results were no more marked.

The apparent absence of a specific character in the papular eruption produced by inoculating the small-pox virus, might aave led to the belief that it was merely inflammation produced at the seat of puncture. For instance, the first animal inoculated was a calf, which was inoculated at the vulva. A number of small papules appeared, and it was imagined that a very characteristic eruption of cow-pox was about to be developed; but instead of developing into pustules, they rapidly disappeared without leaving any trace of their existence, and then the result was considered negative, and the animal put to one side. But being desirous, some time afterwards, of producing cow-pox by means of vaccine lymph of Neapolitan origin, this calf, being at hand, was vaccinated from a magnificent pustule raised on another calf which presented a very fine vaccinal eruption. On the eighth day the inoculation had quite failed, greatly to the astonishment and regret of the Commission, as, relying on its success, they had not provided themselves with any more lymph, and had to send for another supply. Thinking the inoculation had not been properly performed, when the new lymph came it was tried again with the utmost care, but another failure resulted in this calf, while with other two animals - a horse and a calf-vaccinated at the same time, there was a beautiful eruption. The Commission were now compelled to admit either that the small-pox inoculation had produced a specific eruption capable of protecting the animal from vaccinia, that it had been previously affected with that disease, or it had no receptivity for the vaccine virus. At that time only about eight bovines had been vaccinated, and it was not certain whether this species would contract the cow-pox in this way. The experiments were continued, altogether ten animals were variolised, and then vaccinated some days afterwards; but six had no vaccinal eruption whatever, three had rudimentary and ephemeral pustules, and one only showed a regular and characteristic cow-pox. Considering the readiness, as was afterwards found, with which true cow-pox, human vaccinia, and horse-pox could be inoculated, and that the former could only be produced very exceptionally when the animals had been previously variolised, the conclusion arrived at by this important chance discovery was that variolisation exercises a neutralising influence on the development of vaccinia; and that the slightly-marked eruption, so undecided in character, caused by the inoculation of small-pox virus on bovines, is of a specific naturein this species presenting, with the cow-pox, the same relations that variola and vaccinia do to each other in the human species.

(To be continued.)

\section{SUDDEN AND UNEXPECTED DEATH FROM RUPTURE OF A VESSEL IN THE CERVICAL PORTION OF THE SPINAL CORD.}

BY FREDERICK PAGE, M.D. EDIN.,

LECTURER ON MEDICAL JURISPRUDENCE IN THE UNIVERSITY OF DURHAM, AND SURGEON TO THE HOSPITAL FOR SICK CHILDREN, NEWCASTLE-UPON-TYNE.

DURING an indisposition of some five days' duration, a delicate girl, aged nine years and eleven months, suddenly died, as an eye-witness described, " choking." The history of the case is very imperfect. The child's stepmother was a most peculiar woman, from whom no reliable information could be obtained, and the father saw little of the child in consequence of his being away from home all day, and often for part of the night. The girl was a seven months' child, and had been delicate from birth, though she had no medical advice for eleven months previnus to her death. For a considerable time she had suffered at irregular intervals from attacks of indisposition, lasting some few days, and leaving her debilitated, but otherwise in her usual health. During these attacks she always complained of stiffness of the neck, of pain, sometimes very severe, along the upper part of the spine, and she kept her left arm still because she felt pain on moving it. She was prostrate, and alternately hot and cold. She did not vomit, never had convulsions, nor any kind of fit. On a Saturday she was in her usual health, and took a long walk. On Sunday she was ailing. On Monday she stayed away from school, and was confined to the house, complaining of pain and stiffness in the neck and $\mathrm{e}^{\mathrm{ft}}$ shoulder. She refused her food, and was thirsty. She was much the same on Tuesday. On Wednesday afternoon she was put to bed, and on Thursday morning she seemed to be much better. At 4 P.M., just after her bowels had been moved, she suddenly died without being seen by a medical man.

Autopsy, twenty-four hours after death. -The body was thin, but not emaciated. The muscles of the arms were much less plump than those of the legs. Froth was issuing from the mouth. There were no marks of injury. Nothing was found to account for death in the belly, chest, or skull. In the substance of the spinal cord, at the lower part of the cervical region, a considerable infiltration of blood was found, and on section a clot about the size of a horse-bean escaped. The blood was infiltrated into the anterior and lateral tracts of the cord more than into the posterior portion. The cord around the infiltration was soft. The membranes and the vertebro appeared to be quite healthy. There can be little doubt, I think, that this child was the subject of chronic disease of the spinal cord, which gave rise to the peculiar periodic attacks from which she suffered, and that the immediate cause of death was paralysis of the muscles of respiration from pressure upon the origin of the phrenic and other nerves. The blood proceeded from a vessel in the cord, ruptured, probably, by the effort of emptying the bowels.

The causes of death, and particularly of unexpected deaths, are well worthy the most careful consideration; and my object in placing the above case upon record is more to direct attention to a rare and peculiar explanation of sudden death than to enlarge upon the many features of interest which I think the case cannot fail to possess for the physician and the pathologist.

\section{A CASE OF INTERFERENCE WITH NAIL- GROWTH DURING ILLNESS.}

\section{By GEORGE THIN, M.D.}

IN the volume of Lectures on Dermatology delivered in the Royal College of Surgeons of England in 1876-77-78, by Mr. Erasmus Wilson, reference is made (p. 78) to cases reported by Dr. Beau, of arrested nail-formation associated with illness. The publication of a certain number of cases in which the dates of appearance and disappearance of the depression in the nails are authentic may be useful, and I therefore give the following details regarding one that has come under my notice.

A middle-aged man was seized with severe colic-pains in the end of August, was confined to bed for a week, during which time he ate very little and suffered much from retching. It was only at the end of the third week that he regained his usual appetite.

Shortly afterwards I had occasion to observe that in every nail of both hands the groove described by Dr. Beau was to be plainly seen-a well-marked depression nearly a millimetre broad extending across the nails. When I first saw the depressions they had advanced nearly to the middle of the nails. I observed their progress forwards with interest, and found that they had not completely disappeared at the points of the nails until the first week of January.

Thus, from the date of the illness until the final disappearance of the corresponding depression in the nails there was a period of four months, and this is the fact which $I$ think is worthy of being put on record.

Queen Anne-street, W.

\section{O N T O N G A.}

\section{BY C. BADER,}

OPHTHALMIC SURGEON TO GUY'S HOSPITAL

THE results obtained from Tonga by Drs. Ringer and Murrell (see The LANCET, March 6th, page 360) fully coincide with mine. I have notes of cases of brain and kidney disease in which tonga alone succeeded in removing pain. I shall, however, confine myself to reporting the effects upon the eye. Some months ago, when commencing 